\title{
Variations in desiccation tolerance in seeds of Eugenia pyriformis: dispersal at different stages of maturation ${ }^{1}$
}

\author{
Variações da tolerância à dessecação em sementes de Eugenia pyriformis: dispersão \\ em diferentes estádios de maturação
}

\author{
Edmir Vicente Lamarca ${ }^{2 *}$, Marcelo Bento Paes de Camargo ${ }^{3}$, Simone de Padua Teixeira ${ }^{4}$, Edvaldo Aparecido \\ Amaral da Silva ${ }^{5}$, Jose Marcio Rocha Faria ${ }^{6}$ e Claudio Jose Barbedo ${ }^{7}$
}

\begin{abstract}
Eugenia pyriformis Cambess., known locally as uvaieira, a species of fruit-bearing tree with both pharmacological and gastronomic potential, has seeds which are sensitive to desiccation. The aim of this study was to analyse whether the degree of tolerance to desiccation of uvaieira seeds depends on the stage of maturation of the seeds at shedding. This, in turn, depends on the environmental conditions in which the seeds develop, including the accumulation of degree-days and rainfall in the period. Seeds were collected from the ripe fruit of parent plants located in the states of São Paulo and Minas Gerais, Brazil, submitted to drying and analysed for water content and germination. A completely randomised design was used in a $20 \times 3$ factorial scheme (source of material $\mathrm{x}$ level of drying). The degree of desiccation tolerance differs between region and period of collection, even for the same parent plant when the seeds are collected in different years. The water and thermal conditions of the environment during seed development modify the maturation cycle, the physiological quality and the acquisition of desiccation tolerance. In uvaieira seeds, desiccation tolerance depends on the physiological maturity of the seeds at the time of dispersal, which is associated with the environmental conditions.
\end{abstract}

Key words: Myrtaceae. Uvaieira. Degree days. Physiological quality. Drying.

\begin{abstract}
RESUMO - A uvaieira (Eugenia pyriformis Cambess.), espécie arbórea e frutífera, com potencial farmacológico e gastronômico, apresenta sementes sensíveis à dessecação. O objetivo deste trabalho foi analisar se o grau de tolerância à dessecação de sementes de uvaieira depende do grau de maturidade das sementes no momento de sua dispersão. Este, por sua vez, depende das condições ambientais nas quais as sementes se desenvolvem, incluindo-se o acúmulo de graus-dia e a chuva acumulada no período. As sementes foram coletadas de frutos maduros de matrizes localizadas em São Paulo e Minas Gerais, submetidas à secagem e analisadas quanto ao teor de água e germinação. Utilizou-se o delineamento inteiramente casualizado, em esquema fatorial 20 x 3 (origem do material x níveis de secagem). O grau de tolerância à dessecação difere entre as regiões e épocas de coleta, inclusive dentro da mesma matriz, quando as sementes são coletadas em anos distintos. As condições hídricas e térmicas do ambiente, durante o desenvolvimento das sementes, condicionam o ciclo de maturação, a qualidade fisiológica e a aquisição da tolerância à dessecação. A tolerância à dessecação de sementes de uvaieira depende da maturidade fisiológica das sementes no momento de sua dispersão, a qual está associada às condições ambientais.
\end{abstract}

Palavras-chave: Myrtaceae. Uvaieira. Graus-dia. Qualidade fisiológica. Secagem.

\footnotetext{
DOI: $10.5935 / 1806-6690.20160014$

* Autor para correspondência

${ }^{1}$ Recebido para publicação em 11/08/2014; aprovado em 21/09/2015

Parte da Tese do primeiro autor apresentada no Programa de Pós-Graduação em Biodiversidade Vegetal e Meio Ambiente/Instituto de Botânica; apoio financeiro e bolsas de estudo: CNPq e CAPES

${ }^{2}$ Programa de Pós-Graduação em Biodiversidade Vegetal e Meio Ambiente, Instituto de Botânica, São Paulo-SP, Brasil, edmirvicente18@ gmail.com ${ }^{3}$ Centro de Ecofisiologia e Biofísica, Climatologia Agrícola, Instituto Agronômico de Campinas, Campinas-SP, Brasil, mcamargo@ iac.sp.gov.br ${ }^{4}$ Faculdade de Ciências Farmacêuticas de Ribeirão Preto, Universidade de São Paulo, Ribeirão Preto-SP, Brasil, spadua@ @cfrp.usp.br ${ }^{5}$ Faculdade de Ciências Agronômicas, Universidade Estadual Paulista, Botucatu-SP, Brasil, amaraldasilva@fca.unesp.br ${ }^{6}$ Departamento de Ciências Florestais, Universidade Federal de Lavras, Lavras-MG, Brasil, jmfaria@dcf.ufla.br

${ }^{7}$ Núcleo de Pesquisa em Sementes, Instituto de Botânica, São Paulo-SP, Brasil, claudio.barbedo@pesquisador.cnpq.br
} 


\section{INTRODUCTION}

Known locally as uvaieira, Eugenia pyriformis Cambess. is a species of fruit-bearing tree with great gastronomic, industrial and medicinal potential; besides being appreciated for its fresh fruit, it is also widely used in agroforestry systems (LAMARCA et al., 2013b). It belongs to the family Myrtaceae, and occurs in tropical or subtropical regions of Argentina, Paraguay and Brazil. The seeds are intolerant to desiccation and short-term storage (DELGADO; BARBEDO, 2012; SCALON et al., 2012), with extensive germination plasticity (AMADOR; BARBEDO, 2011).

Seeds are crucial to most processes of crop production, both for their use directly in the field and in the production of seedlings. The vast majority have an exceptional capacity for storage, maintaining germination potential for several years and allowing their use at completely different times and places from those where they were produced. However, there is a group of seeds that, being sensitive to desiccation, can only be stored for extremely short periods, often preventing their use in production systems (ARAÚJO et al., 2008; HAY; PROBERT, 2013).

Sensitivity to desiccation varies between species, ranging from the more sensitive to the more tolerant (PEREZ; HILL; WALTERS, 2012), with variations occurring within a single species, and depending on the environmental conditions in which the seeds are formed (DAWS et al., 2006; DUSSERT et al., 2000; JOËT; OURCIVAL; DUSSERT, 2013; LAMARCA et al., 2013a.). This led to the assumption that changes in the desiccation tolerance of seeds may be due to variations in their degree of physiological maturity when dispersed (BARBEDO; CENTENO; FIGUEIREDO-RIBEIRO, 2013).

Development of the seed, which takes place from fertilisation of the ovule to maturity, is divided into three stages, stage I being marked by cell division and expansion, phase II by the accumulation of reserves and a progressive increase in dry matter, and phase III by drying at maturation, or desiccation. The transition from the second to the third stage roughly coincides with the development of desiccation tolerance (ANGELOVICI et al., 2010).

Physiological evidence suggests that recalcitrant seeds do not complete the second phase; with fruit abscission occurring before development is complete (DAWS et al., 2004; DELGADO; BARBEDO, 2012). However, studies with desiccation-intolerant seeds (Aesculus hippocastanum and Acer pseudoplatanus), collected at different locations in Europe, have shown that variations in air temperature, represented by the concept of degree-days, influence the physiological maturity of seeds, affecting acquisition of desiccation tolerance (DAWS et al., 2004, 2006). In seeds of Euterpe edulis and Tapirira obtuse, for example, variations have been seen in desiccation tolerance and storage, depending on the environmental conditions in which the seeds were formed (MARTINS et al., 2009; PEREIRA et al., 2012).

As it has seeds that are desiccation-intolerant and of short-term storage, and as productive individuals are found over a wide range of latitudes and altitudes, the uvaieira is an interesting model for the physiological study of seeds. Analysis of the germination behaviour of seeds from different regions can provide important information about the degree of environmental interference on the characteristics of formed seeds. In this study, the hypothesis was considered and tested, that the level of desiccation tolerance in seeds of the uvaieira depends on the degree of maturity reached by the seeds at the time of dispersal. This in turn depends on the environmental conditions in which the seeds develop, including the accumulation of degree-days, and accumulated rainfall for the period.

\section{MATERIAL AND METHODS}

Seeds of the uvaieira were obtained from mature, recently dispersed fruits, based on information on maturation and dispersal from Lamarca et al. (2013a) and Teixeira and Barbedo (2012). The fruits came from 11 different regions, as described in Table 1. Codes were allocated for region, and period or altitude, as follows: Lavras (LAV), São Bento do Sapucaí (SBS), Campinas (CAM) São Paulo (SPA), Ibiúna (IBI), São Bernardo do Campo (SBC), Ribeirão Preto (RIB), Jumirim (JUM), Santo André (SAA), Itaberá (ITA) and Pariquera-Açu (PAR). In five of these regions (LAV, CAM, SPA, IBI, and SBC), seeds were obtained in different years from the same parent plants, identified by number (1, 2 and 3), and in SBS from parent plants at three different altitudes, also identified by number. Each collection, considering region and period, or region and altitude, was regarded as a different source of seed, also referred to as source of material.

During the period of maximum flowering for the species, the inflorescences of those trees with most flowers at anthesis were marked at each source. At the end of the period of fruit development and ripening, the mature, recently dispersed fruits were collected (up to 24 hours after dispersal), from which the seeds were manually extracted (TEIXEIRA; BARBEDO, 2012) and stored in a cold room at $7{ }^{\circ} \mathrm{C}$ until the start of the experiments 
Table 1 - Source of uvaieira seeds (Eugenia pyriformis Cambess.), maturation cycle (time between flowering and dispersal), meteorological data of the collection sites during the cycle of seed formation, and physical characteristics of the collected material

\begin{tabular}{|c|c|c|c|c|c|c|}
\hline Source of seed & $\begin{array}{l}\text { Maturation cycle } \\
\text { (total of cycle) }\end{array}$ & $\begin{array}{l}\text { Min- Max } \\
\quad\left({ }^{\circ} \mathrm{C}\right)\end{array}$ & $\begin{array}{c}\mathrm{DD} \\
\left({ }^{\circ} \mathrm{C} \text { day }\right)\end{array}$ & $\begin{array}{l}\text { Rainfall } \\
\text { (mm) }\end{array}$ & $\mathrm{WC}\left(\mathrm{g} \mathrm{g}^{-1}\right)$ & $\begin{array}{c}\mathrm{DM} \\
\left(\mathrm{g} \mathrm{seed}^{-1}\right)\end{array}$ \\
\hline $\begin{array}{l}\text { LAV1 - Lavras, MG (21 }{ }^{\circ} 13^{\prime} \mathrm{S}, \\
44^{\circ} 58^{\prime} \mathrm{W}, 949 \mathrm{~m} \text {; Cwa) }\end{array}$ & $\begin{array}{c}09 / 08 / 09- \\
23 / 09 / 09 \text { (45 days) }\end{array}$ & $15-27$ & 495 & 175.4 & $1.53 \pm 0.10$ & $0.59 \pm 0.03$ \\
\hline $\begin{array}{l}\text { LAV2 - Lavras, MG }\left(21^{\circ} 13^{\prime} \mathrm{S},\right. \\
\left.44^{\circ} 58^{\prime} \mathrm{W}, 949 \mathrm{~m} \text {; Cwa }\right)\end{array}$ & $\begin{array}{c}15 / 08 / 10- \\
25 / 09 / 10(41 \text { days })\end{array}$ & $12-28$ & 417 & 24.2 & $1.12 \pm 0.06$ & $0.80 \pm 0.08$ \\
\hline $\begin{array}{l}\text { SBS1 - São Bento do Sapucaí, SP } \\
\left(22^{\circ} 41^{\prime} \mathrm{S}, 45^{\circ} 43^{\prime} \mathrm{W}, 884 \mathrm{~m} \text {; Cfb) }\right.\end{array}$ & $\begin{array}{c}24 / 08 / 10- \\
07 / 10 / 10(44 \text { days })\end{array}$ & $12-27$ & 426 & 177.1 & $1.87 \pm 0.24$ & $0.37 \pm 0.06$ \\
\hline $\begin{array}{l}\text { SBS2 - São Bento do Sapucaí, SP } \\
\left(22^{\circ} 41^{\prime} \mathrm{S}, 45^{\circ} 45^{\prime} \mathrm{W}, 1022 \mathrm{~m} \text {; Cfb }\right)\end{array}$ & $\begin{array}{c}26 / 08 / 10- \\
15 / 10 / 10(50 \text { days })\end{array}$ & $11-26$ & 448 & 177.1 & $1.34 \pm 0.11$ & $0.97 \pm 0.07$ \\
\hline $\begin{array}{l}\text { SBS3 - São Bento do Sapucaí, SP } \\
\left(22^{\circ} 41^{\prime} \text { S, } 45^{\circ} 46^{\prime} \text { W, } 1121 \text { m; Cfb }\right)\end{array}$ & $\begin{array}{c}26 / 08 / 10- \\
21 / 10 / 10 \text { (56 days) }\end{array}$ & $11-26$ & 472 & 252.4 & $1.12 \pm 0.04$ & $1.27 \pm 0.04$ \\
\hline $\begin{array}{l}\text { CAM1 - Campinas, SP }\left(22^{\circ} 52^{\prime} \mathrm{S} \text {, }\right. \\
47^{\circ} 04^{\prime} \mathrm{W}, 645 \mathrm{~m} \text {; Cwa) }\end{array}$ & $\begin{array}{c}01 / 08 / 09- \\
18 / 09 / 09 \text { (48 days) }\end{array}$ & $14-26$ & 479 & 162.7 & $1.58 \pm 0.08$ & $0.31 \pm 0.03$ \\
\hline $\begin{array}{l}\text { CAM2 - Campinas, SP }\left(22^{\circ} 52^{\prime} \mathrm{S} \text {, }\right. \\
47^{\circ} 04^{\prime} \mathrm{W}, 645 \mathrm{~m} \text {; Cwa) }\end{array}$ & $\begin{array}{c}10 / 08 / 10- \\
19 / 09 / 10 \text { (40 days) }\end{array}$ & $14-28$ & 451 & 6.3 & $1.19 \pm 0.11$ & $0.48 \pm 0.05$ \\
\hline $\begin{array}{l}\text { CAM3 - Campinas, SP (22॰52’ S, } \\
47^{\circ} 04^{\prime} \mathrm{W}, 645 \mathrm{~m} \text {; Cwa) }\end{array}$ & $\begin{array}{c}\text { 06/08/11 - } \\
14 / 09 / 11 \text { (39 days) }\end{array}$ & $14-28$ & 442 & 36.7 & $1.44 \pm 0.08$ & $0.52 \pm 0.04$ \\
\hline $\begin{array}{l}\text { SPA1 - São Paulo, SP }\left(23^{\circ} 38^{\prime} \mathrm{S} \text {, }\right. \\
46^{\circ} 37^{\prime} \mathrm{W}, 785 \mathrm{~m} \text {; Cwb) }\end{array}$ & $\begin{array}{c}25 / 08 / 09- \\
12 / 10 / 09 \text { (48 days) }\end{array}$ & $15-24$ & 458 & 232.1 & $2.16 \pm 0.44$ & $0.19 \pm 0.04$ \\
\hline $\begin{array}{l}\text { SPA2 - São Paulo, SP }\left(23^{\circ} 38^{\prime} \mathrm{S} \text {, }\right. \\
\left.46^{\circ} 37^{\prime} \mathrm{W}, 785 \mathrm{~m} ; \mathrm{Cwb}\right)\end{array}$ & $\begin{array}{c}26 / 08 / 10- \\
09 / 10 / 10 \text { (44 days) }\end{array}$ & $14-25$ & 428 & 128.8 & $1.82 \pm 0.15$ & $0.23 \pm 0.03$ \\
\hline $\begin{array}{l}\text { SPA3 - São Paulo, SP }\left(23^{\circ} 38^{\prime} \mathrm{S} \text {, }\right. \\
46^{\circ} 37^{\prime} \mathrm{W}, 785 \mathrm{~m} \text {; Cwb) }\end{array}$ & $\begin{array}{c}31 / 08 / 11- \\
10 / 10 / 11 \text { (40 days) }\end{array}$ & $13-25$ & 374 & 99.1 & $1.90 \pm 0.14$ & $0.24 \pm 0.02$ \\
\hline $\begin{array}{l}\text { IBI1 - Ibiúna, SP }\left(23^{\circ} 39^{\prime} \text { S, } 47^{\circ} 09^{\prime} \mathrm{W} \text {, }\right. \\
917 \text { m; Cfb) }\end{array}$ & $\begin{array}{c}12 / 09 / 10- \\
23 / 10 / 10(41 \text { days })\end{array}$ & $12-27$ & 413 & 123.6 & $1.53 \pm 0.11$ & $0.38 \pm 0.05$ \\
\hline $\begin{array}{l}\text { IBI2 - Ibiúna, SP }\left(23^{\circ} 39^{\prime} \text { S, } 47^{\circ} 09^{\prime} \mathrm{W} \text {, }\right. \\
917 \text { m; Cfb) }\end{array}$ & $\begin{array}{c}04 / 09 / 11- \\
12 / 10 / 11 \text { (38 days) }\end{array}$ & $10-29$ & 380 & 103.2 & $1.71 \pm 0.18$ & $0.19 \pm 0.03$ \\
\hline $\begin{array}{l}\text { SBC1 - São Bernardo do Campo, SP } \\
\left(23^{\circ} 42^{\prime} \mathrm{S}, 46^{\circ} 33^{\prime} \mathrm{W}, 786 \mathrm{~m} \text {; Cwb) }\right.\end{array}$ & $\begin{array}{c}16 / 08 / 10- \\
03 / 10 / 10 \text { (48 days) }\end{array}$ & $13-25$ & 458 & 104.3 & $1.27 \pm 0.07$ & $0.55 \pm 0.03$ \\
\hline $\begin{array}{l}\text { SBC2 - São Bernardo do Campo, SP } \\
\left(23^{\circ} 42^{\prime} \mathrm{S}, 46^{\circ} 33^{\prime} \mathrm{W}, 786 \mathrm{~m} \text {; Cwb) }\right.\end{array}$ & $\begin{array}{c}16 / 08 / 11- \\
01 / 10 / 11 \text { (46 days) }\end{array}$ & $13-25$ & 406 & 68.5 & $1.38 \pm 0.10$ & $0.39 \pm 0.02$ \\
\hline $\begin{array}{l}\text { SAA - Santo André, SP (2340’ S, } \\
46^{\circ} 32^{\prime} \mathrm{W}, 791 \mathrm{~m} \text {; Cwb) }\end{array}$ & $\begin{array}{c}\text { 14/08/07 - } \\
\text { 27/09/07 (44 days) }\end{array}$ & $13-26$ & 431 & 3.2 & $1.56 \pm 0.07$ & $0.48 \pm 0.05$ \\
\hline $\begin{array}{l}\text { JUM - Jumirim, SP }\left(22^{\circ} 05^{\prime} \mathrm{S},\right. \\
\left.47^{\circ} 47^{\prime} \mathrm{W}, 540 \mathrm{~m} \text {; Cwa }\right)\end{array}$ & $\begin{array}{c}\text { 05/08/10 - } \\
19 / 09 / 10 \text { (45 days) }\end{array}$ & $12-29$ & 481 & 12.6 & $1.47 \pm 0.09$ & $0.43 \pm 0.06$ \\
\hline $\begin{array}{l}\text { RIB - Ribeirão Preto, SP ( } 21^{\circ} 10^{\prime} \mathrm{S} \text {, } \\
47^{\circ} 52^{\prime} \mathrm{W}, 593 \mathrm{~m} \text {; Cwa) }\end{array}$ & $\begin{array}{c}14 / 08 / 10- \\
17 / 09 / 10 \text { (34 days) }\end{array}$ & $14-31$ & 440 & 6.9 & $1.70 \pm 0.08$ & $0.48 \pm 0.04$ \\
\hline $\begin{array}{l}\text { ITA - Itaberá, SP }\left(23^{\circ} 52^{\prime} \mathrm{S}, 49^{\circ} 06^{\prime} \mathrm{W} \text {, }\right. \\
683 \text { m; Cfa) }\end{array}$ & $\begin{array}{c}17 / 08 / 10- \\
23 / 09 / 10 \text { (37 days) }\end{array}$ & $11-26$ & 341 & 1.8 & $1.93 \pm 0.22$ & $0.42 \pm 0.02$ \\
\hline $\begin{array}{l}\text { PAR - Pariquera-Açú, SP }\left(24^{\circ} 37^{\prime} \text { S, }\right. \\
\left.47^{\circ} 53^{\prime} \text { W, } 28 \text { m; Af }\right)\end{array}$ & $\begin{array}{c}23 / 08 / 10- \\
11 / 10 / 10(49 \text { days })\end{array}$ & $15-25$ & 501 & 87.4 & $1.49 \pm 0.13$ & $0.67 \pm 0.02$ \\
\hline
\end{tabular}

Min: minimum temperature; Max: maximum temperature; DD: Degree-days; Rainfall: accumulated rainfall for the period. Water content (WC) and dry matter $(\mathrm{DM})$ : mean values \pm standard deviation. Köppen climate classification: $\mathrm{Cwa}$ - temperate humid climate with dry winters and hot summers; Cwb - temperate humid climate with dry winters and temperate summers; Cfa - subtropical climate; Cfb - maritime climate; Af - equatorial climate or tropical humid 
(DELGADO; BARBEDO, 2007), but not for more than seven days after collection.

From weather stations located close to the collection areas, data were obtained of the daily rainfall $(\mathrm{mm})$ and the maximum and minimum air temperature $\left({ }^{\circ} \mathrm{C}\right)$. The accumulated rainfall $(\mathrm{mm})$ and degree-days $\left({ }^{\circ} \mathrm{C}\right.$ day) were calculated for the period between flowering and seed dispersal, as per equations proposed by Villa Nova et al. (1972), considering a basal temperature of $10{ }^{\circ} \mathrm{C}$ (PEDRO JUNIOR et al., 1977).

After collection, the seeds were characterised for water content, dry matter content and germination. The water content and dry matter content were determined gravimetrically by oven method at $103{ }^{\circ} \mathrm{C}$ for 17 hours (BRASIL, 2009), the results being shown as $\mathrm{g}$ of water per $g$ of dry matter $\left(\mathrm{g} \mathrm{g}^{-1}\right)$ for water content, and $\mathrm{g} \mathrm{seed}^{-1}$ for dry matter content.

Germination tests were carried out in germination chambers at $25{ }^{\circ} \mathrm{C}$ under constant light and $100 \%$ relative humidity, using roll paper as substrate (filter paper), with two sheets for the base and one as cover (BRASIL, 2009). Germination was evaluated every 3 days for 70 days (DELGADO; BARBEDO, 2007; LAMARCA; SILVA; BARBEDO, 2011), recording those seeds with a primary root, to calculate germinated seeds, and those that had produced normal seedlings, to calculate germination; both results were given as a percentage. The germination speed index (GSI) was also calculated, using the formula proposed by Maguire (1962).

After characterisation, the seeds underwent controlled drying in a forced air circulation oven; for this, they were placed on shelves lined with a single layer of polyethylene screen, with no overlaps. Drying was carried out intermittently, with 10 hours at $40{ }^{\circ} \mathrm{C}$ followed by 14 hours at $20-25{ }^{\circ} \mathrm{C}$ (DELGADO; BARBEDO, 2007). Periodically, based on the value for seed dry matter, samples were taken and evaluated for water content, dry matter content and germination, as described above. This procedure was repeated until the seeds reached two pre-established levels, $1.00 \mathrm{~g} \mathrm{~g}^{-1}$ and $0.67 \mathrm{~g} \mathrm{~g}^{-1}$, i.e. when $50 \%$ of the vigour and germination are lost respectively (DELGADO; BARBEDO, 2007). Levels of drying were thus created: non-dried seeds $\left(D_{0}\right)$, seeds dried to the first level $\left(\mathrm{D}_{\mathrm{I}}\right)$ and seeds dried to the second level $\left(\mathrm{D}_{\mathrm{II}}\right)$.

The experimental design was completely randomised, in a $20 \times 3$ factorial scheme (source of material $x$ level of drying), with three replications. The resulting data were submitted to variance analysis $(\mathrm{F}$ test) at a significance level of 5\%. There was no need for data transformation, and mean values were compared by Tukey's test at the 5\% significance level (SANTANA; RANAL, 2004). Simple correlation coefficients were then calculated for all combinations of the meteorological data with the physical and physiological data of the seeds, and the significance determined by t-test at $5 \%$ probability.

\section{RESULTS AND DISCUSSION}

Analysis of variance (Table 2) showed a significant interaction between the factors source of material and level of seed drying, for the data on germinated seeds, germination and GSI, with a difference in the desiccation tolerance of the seeds for source therefore becoming evident.

The increase in drying decreased the values for germinated seeds (Table 3), germination (Table 4) and SGI (Table 5). It was also found that, depending on the source of the material, this decrease occurred at the first or second level of drying (in some cases, at both), showing that there were differences in the desiccation tolerance of the seeds. Thus seeds coming from LAV1, SBS1, CAM1, CAM3, SPA1, SPA3, IBI1, SAA and PAR showed a reduction for all physiological variables shortly after the first level of drying, unlike the seeds

Table 2 - Summary of analysis of variance for the variables germinated seeds (GS), germination (G) and germination speed index (GSI) in the uvaieira (Eugenia pyriformis Cambess.) from different sources and at three levels of drying

\begin{tabular}{|c|c|c|c|c|c|c|c|}
\hline \multirow{2}{*}{ Cause of variation } & \multirow{2}{*}{$\mathrm{DF}$} & \multicolumn{2}{|c|}{------------GS-------------- } & \multicolumn{2}{|c|}{----------------G----------------- } & \multicolumn{2}{|c|}{------------GSI------------- } \\
\hline & & MS & $\mathrm{F}$ & MS & $\mathrm{F}$ & MS & $\mathrm{F}$ \\
\hline Source of material (A) & 19 & 938.905 & $12.3^{*}$ & 1845.046 & $28.5^{*}$ & 0.416 & $91.5^{*}$ \\
\hline Level of drying (B) & 2 & 34661.239 & $452.8^{*}$ & 42953.867 & $663.6^{*}$ & 6.392 & $1406.6^{*}$ \\
\hline $\mathrm{A} \times \mathrm{B}$ & 38 & 573.578 & $7.5^{*}$ & 818.884 & $12.7 *$ & 0.127 & $27.9 *$ \\
\hline Residual error & 120 & 76.544 & & 64.733 & & 0.004 & \\
\hline $\mathrm{CV}(\%)$ & & \multicolumn{2}{|c|}{12.10} & \multicolumn{2}{|c|}{14.35} & \multicolumn{2}{|c|}{12.06} \\
\hline
\end{tabular}


from RIB, LAV2, SBS2, SBS3, CAM2, JUM, SPA2, IBI2, SBC1, SBC2 and ITA, which showed a reduction after the second level (Tables 3, 4 and 5).

When each variable is considered individually, it is found that the differences in desiccation tolerance occurred even when comparing seeds from the same region but from different altitudes or periods, for example germinated seeds from SBS, LAV, CAM SPA, IBI and SBC (Table 3).

These results support the idea that desiccation tolerance is not established absolutely, but rather in a continuous process, and may vary not only inter- but also intra-species (WALTERS, 2000; DAWS et al., 2004; DELGADO; BARBEDO, 2012; PEREZ; HILL, WALTERS, 2012). These differences had already been seen in seeds of various species of Eugenia, among which E. pyriformis is among the most sensitive to desiccation (DELGADO; BARBEDO, 2007). However, there were still no studies into the species, where production years and places of origin were varied.

As mentioned above, the desiccation tolerance of the seeds varied with both region and year of production, showing the influence of environmental conditions on this characteristic. Data of the maturation cycle (which varied from 34 to 56 days), accumulated degree-days (341 to 501 degree-days), maximum air temperature $\left(24\right.$ to $\left.31^{\circ} \mathrm{C}\right)$ and minimum air temperature $\left(10\right.$ to $\left.15^{\circ} \mathrm{C}\right)$, accumulated rainfall in the period ( 1.8 to $252.4 \mathrm{~mm}$ ), water content (1.12 to $2.16 \mathrm{~g} \mathrm{~g}^{-1}$ ) and seed dry matter content (1.27 to $\left.0.19 \mathrm{~g} \mathrm{seed}^{-1}\right)$, for the period of development/maturation (Table 1), showed wide variation for the different regions and years of production. It was found that years/regions with greater amounts of accumulated rainfall or a greater accumulation of degree-days resulted in the longest maturation cycles (Table 1), moreover with a positive correlation (Table 6), suggesting that those variables

Table 3 - Germinated seeds of the uvaieira (Eugenia pyriformis Cambess.) from different sources and at three levels of drying. $\mathrm{D}_{0}$ - non-dried seeds; $\mathrm{D}_{\mathrm{I}}-1$ st level of drying; $\mathrm{D}_{\mathrm{II}}-2$ nd level of drying

\begin{tabular}{|c|c|c|c|}
\hline \multirow{2}{*}{$\begin{array}{l}\text { Source of material } \\
\text { (Water content for level of drying, } \mathrm{g} \mathrm{g}^{-1} \text { ) }\end{array}$} & \multicolumn{3}{|c|}{ Level of drying* } \\
\hline & $\mathrm{D}_{0}$ & $\mathrm{D}_{\mathrm{I}}$ & $\mathrm{D}_{\mathrm{II}}$ \\
\hline $\operatorname{LAV} 1\left(\mathrm{D}_{0}=1.53 ; \mathrm{D}_{\mathrm{I}}=1.00 ; \mathrm{D}_{\mathrm{II}}=0.70\right)$ & $100 \mathrm{aA}$ & 77 abcdeB & 33 defC \\
\hline $\operatorname{LAV} 2\left(\mathrm{D}_{0}=1.12 ; \mathrm{D}_{\mathrm{I}}=1.00 ; \mathrm{D}_{\mathrm{II}}=0.67\right)$ & $100 \mathrm{aA}$ & $100 \mathrm{aA}$ & $62 \mathrm{abcB}$ \\
\hline $\operatorname{SBS} 1\left(\mathrm{D}_{0}=1.87 ; \mathrm{D}_{\mathrm{I}}=1.17 ; \mathrm{D}_{\mathrm{II}}=0.67\right)$ & $98 \mathrm{abA}$ & 69 bcdeB & $44 \mathrm{bcdeC}$ \\
\hline $\operatorname{SBS} 2\left(\mathrm{D}_{0}=1.34 ; \mathrm{D}_{\mathrm{I}}=1.04 ; \mathrm{D}_{\mathrm{II}}=0.64\right)$ & $97 \mathrm{abA}$ & $100 \mathrm{aA}$ & $62 \mathrm{abcB}$ \\
\hline $\operatorname{SBS} 3\left(\mathrm{D}_{0}=1.12 ; \mathrm{D}_{\mathrm{I}}=0.96 ; \mathrm{D}_{\mathrm{II}}=0.64\right)$ & $92 \mathrm{abA}$ & $88 \mathrm{abcA}$ & $52 \mathrm{abcdB}$ \\
\hline $\operatorname{CAM} 1\left(\mathrm{D}_{0}=1.58 ; \mathrm{D}_{\mathrm{I}}=0.96 ; \mathrm{D}_{\mathrm{II}}=0.79\right)$ & $93 \mathrm{abA}$ & 75 abcdeB & $15 \mathrm{fC}$ \\
\hline $\operatorname{CAM} 2\left(D_{0}=1.19 ; D_{I}=0.96 ; D_{I I}=0.64\right)$ & $98 \mathrm{abA}$ & $93 \mathrm{abA}$ & 50 bcdeB \\
\hline $\operatorname{CAM} 3\left(D_{0}=1.44 ; D_{I}=0.96 ; D_{I I}=0.70\right)$ & $93 \mathrm{abA}$ & $53 \mathrm{efB}$ & 25 efC \\
\hline $\operatorname{SPA} 1\left(\mathrm{D}_{0}=2.16 ; \mathrm{D}_{\mathrm{I}}=1.00 ; \mathrm{D}_{\mathrm{II}}=0.75\right)$ & 85 abcA & $52 \mathrm{efB}$ & $38 \operatorname{defB}$ \\
\hline $\operatorname{SPA} 2\left(\mathrm{D}_{0}=1.82 ; \mathrm{D}_{\mathrm{I}}=1.17 ; \mathrm{D}_{\mathrm{II}}=0.67\right)$ & $63 \mathrm{cB}$ & $83 \operatorname{abcdA}$ & $52 \mathrm{abcdB}$ \\
\hline $\operatorname{SPA} 3\left(D_{0}=1.90 ; D_{I}=1.08 ; D_{I I}=0.70\right)$ & $92 \mathrm{abA}$ & 60 defB & 38 cdefC \\
\hline $\operatorname{IBI} 1\left(\mathrm{D}_{0}=1.53 ; \mathrm{D}_{\mathrm{I}}=0.96 ; \mathrm{D}_{\mathrm{II}}=0.64\right)$ & $93 \mathrm{abA}$ & 76 abcdeB & 36 defC \\
\hline $\operatorname{IBI} 2\left(\mathrm{D}_{0}=1.71 ; \mathrm{D}_{\mathrm{I}}=1.22 ; \mathrm{D}_{\mathrm{II}}=0.85\right)$ & 88 abcA & $90 \mathrm{abcA}$ & $62 \mathrm{abcB}$ \\
\hline $\operatorname{SBC} 1\left(\mathrm{D}_{0}=1.27 ; \mathrm{D}_{\mathrm{I}}=1.04 ; \mathrm{D}_{\mathrm{II}}=0.75\right)$ & $73 \mathrm{bcA}$ & 88 abcA & $77 \mathrm{aA}$ \\
\hline $\operatorname{SBC} 2\left(\mathrm{D}_{0}=1.38 ; \mathrm{D}_{\mathrm{I}}=1.08 ; \mathrm{D}_{\mathrm{II}}=0.75\right)$ & $90 \mathrm{abA}$ & $90 \mathrm{abcA}$ & $68 \mathrm{abB}$ \\
\hline $\operatorname{SAA}\left(D_{0}=1.56 ; D_{I}=0.96 ; D_{I I}=0.67\right)$ & $100 \mathrm{aA}$ & $37 \mathrm{fB}$ & 33 defB \\
\hline $\operatorname{JUM}\left(\mathrm{D}_{0}=1.47 ; \mathrm{D}_{\mathrm{I}}=1.00 ; \mathrm{D}_{\mathrm{II}}=0.69\right)$ & $98 \mathrm{abA}$ & $98 \mathrm{aA}$ & 25 efC \\
\hline $\operatorname{RIB}\left(\mathrm{D}_{0}=1.70 ; \mathrm{D}_{\mathrm{I}}=0.96 ; \mathrm{D}_{\mathrm{II}}=0.72\right)$ & $98 \mathrm{abA}$ & $100 \mathrm{aA}$ & $55 \mathrm{abcdB}$ \\
\hline $\operatorname{ITA}\left(\mathrm{D}_{0}=1.93 ; \mathrm{D}_{\mathrm{I}}=1.13 ; \mathrm{D}_{\mathrm{II}}=0.70\right)$ & $100 \mathrm{aA}$ & $100 \mathrm{aA}$ & $42 \mathrm{cdeB}$ \\
\hline $\operatorname{PAR}\left(\mathrm{D}_{0}=1.49 ; \mathrm{D}_{\mathrm{I}}=1.00 ; \mathrm{D}_{\mathrm{II}}=0.85\right)$ & $82 \mathrm{abcA}$ & $65 \mathrm{cdeB}$ & 40 cdefC \\
\hline $\mathrm{CV}(\%)$ & 12.10 & & \\
\hline
\end{tabular}

*Mean values followed by the same letter (lowercase compares source of material and uppercase compares levels of drying) do not differ by Tukey's test at $5 \%$ 
Table 4 - Germination in seeds of the uvaieira (Eugenia pyriformis Cambess.) from different sources and at three levels of drying. $\mathrm{D}_{0}$ - non-dried seeds; $\mathrm{D}_{\mathrm{I}}-1$ st level of drying; $\mathrm{S}_{\mathrm{II}}$ - 2nd level of drying

\begin{tabular}{|c|c|c|c|}
\hline \multirow{2}{*}{$\begin{array}{c}\text { Source of material } \\
\text { (Water content for level of drying, } \mathrm{g} \mathrm{g}^{-1} \text { ) }\end{array}$} & \multicolumn{3}{|c|}{ Level of drying* } \\
\hline & $\mathrm{D}_{0}$ & $\mathrm{D}_{\mathrm{I}}$ & $\mathrm{D}_{\mathrm{II}}$ \\
\hline $\operatorname{LAV} 1\left(\mathrm{D}_{0}=1.53 ; \mathrm{D}_{\mathrm{I}}=1.00 ; \mathrm{D}_{\mathrm{II}}=0.70\right)$ & $98 \mathrm{aA}$ & $47 \mathrm{deB}$ & $10 \mathrm{deC}$ \\
\hline $\operatorname{LAV} 2\left(\mathrm{D}_{0}=1.12 ; \mathrm{D}_{\mathrm{I}}=1.00 ; \mathrm{D}_{\mathrm{II}}=0.67\right)$ & $100 \mathrm{aA}$ & $100 \mathrm{aA}$ & $47 \mathrm{abB}$ \\
\hline $\operatorname{SBS} 1\left(\mathrm{D}_{0}=1.87 ; \mathrm{D}_{\mathrm{I}}=1.17 ; \mathrm{D}_{\mathrm{II}}=0.67\right)$ & $87 \operatorname{abcdA}$ & 38 defB & $18 \mathrm{deC}$ \\
\hline $\operatorname{SBS} 2\left(D_{0}=1.34 ; D_{I}=1.04 ; D_{I I}=0.64\right)$ & 85 abcdA & $90 \mathrm{abA}$ & $42 \mathrm{abcB}$ \\
\hline $\operatorname{SBS} 3\left(\mathrm{D}_{0}=1.12 ; \mathrm{D}_{\mathrm{I}}=0.96 ; \mathrm{D}_{\mathrm{II}}=0.64\right)$ & 77 abcde $\mathrm{A}$ & $77 \mathrm{abA}$ & $32 \mathrm{abcB}$ \\
\hline CAM1 $\left(D_{0}=1.58 ; D_{I}=0.96 ; D_{I I}=0.79\right)$ & 73 bcdeA & $52 \mathrm{cdB}$ & $2 \mathrm{eC}$ \\
\hline $\operatorname{CAM} 2\left(D_{0}=1.19 ; D_{I}=0.96 ; D_{I I}=0.64\right)$ & $90 \mathrm{abcA}$ & $77 \mathrm{abA}$ & $30 \mathrm{bcdB}$ \\
\hline $\operatorname{CAM} 3\left(\mathrm{D}_{0}=1.44 ; \mathrm{D}_{\mathrm{I}}=0.96 ; \mathrm{D}_{\mathrm{II}}=0.70\right)$ & $87 \operatorname{abcdA}$ & 38 defB & $15 \mathrm{deC}$ \\
\hline $\operatorname{SPA} 1\left(D_{0}=2.16 ; D_{I}=1.00 ; D_{I I}=0.75\right)$ & 60 efA & $25 \mathrm{efB}$ & 18 cdeB \\
\hline $\operatorname{SPA} 2\left(D_{0}=1.82 ; D_{I}=1.17 ; D_{I I}=0.67\right)$ & $58 \mathrm{efB}$ & $57 \mathrm{bcA}$ & $42 \mathrm{abcC}$ \\
\hline $\operatorname{SPA} 3\left(D_{0}=1.90 ; D_{I}=1.08 ; D_{I I}=0.70\right)$ & 82 abcdeA & 40 defB & 25 bcdeB \\
\hline $\operatorname{IBI} 1\left(\mathrm{D}_{0}=1.53 ; \mathrm{D}_{\mathrm{I}}=0.96 ; \mathrm{D}_{\mathrm{II}}=0.64\right)$ & 72 cde $\mathrm{A}$ & 33 defB & $18 \mathrm{deB}$ \\
\hline $\operatorname{IBI} 2\left(\mathrm{D}_{0}=1.71 ; \mathrm{D}_{\mathrm{I}}=1.22 ; \mathrm{D}_{\mathrm{II}}=0.85\right)$ & $47 \mathrm{fA}$ & $37 \operatorname{def} A B$ & $23 \mathrm{bcdeB}$ \\
\hline $\operatorname{SBC} 1\left(\mathrm{D}_{0}=1.27 ; \mathrm{D}_{\mathrm{I}}=1.04 ; \mathrm{D}_{\mathrm{II}}=0.75\right)$ & $65 \operatorname{def} A$ & $78 \mathrm{abA}$ & $63 \mathrm{aA}$ \\
\hline $\operatorname{SBC} 2\left(\mathrm{D}_{0}=1.38 ; \mathrm{D}_{\mathrm{I}}=1.08 ; \mathrm{D}_{\mathrm{II}}=0.75\right)$ & 88 abcde $\mathrm{A}$ & 40 defB & 25 bcdeB \\
\hline $\operatorname{SAA}\left(D_{0}=1.56 ; D_{I}=0.96 ; D_{I I}=0.67\right)$ & $97 \mathrm{abA}$ & $17 \mathrm{fB}$ & 23 bcdeB \\
\hline $\mathrm{JUM}\left(\mathrm{D}_{0}=1.47 ; \mathrm{D}_{\mathrm{I}}=1.00 ; \mathrm{D}_{\mathrm{II}}=0.69\right)$ & 92 abc $\mathrm{A}$ & $93 \mathrm{abA}$ & $15 \mathrm{deB}$ \\
\hline $\operatorname{RIB}\left(\mathrm{D}_{0}=1.70 ; \mathrm{D}_{\mathrm{I}}=0.96 ; \mathrm{D}_{\mathrm{II}}=0.72\right)$ & 88 abcdA & $90 \mathrm{abA}$ & $42 \mathrm{abcB}$ \\
\hline $\operatorname{ITA}\left(\mathrm{D}_{0}=1.93 ; \mathrm{D}_{\mathrm{I}}=1.13 ; \mathrm{D}_{\mathrm{II}}=0.70\right)$ & 87 abcdA & $88 \mathrm{abA}$ & 23 bcdeB \\
\hline $\operatorname{PAR}\left(\mathrm{D}_{0}=1.49 ; \mathrm{D}_{\mathrm{I}}=1.00 ; \mathrm{D}_{\mathrm{II}}=0.85\right)$ & $74 \mathrm{bcdeA}$ & 31 defB & $18 \mathrm{deB}$ \\
\hline $\mathrm{CV}(\%)$ & & 14.35 & \\
\hline
\end{tabular}

*Mean values followed by the same letter (lowercase compares source of material and uppercase compares levels of drying) do not differ by Tukey's test at 5\%

Table 5 - Germination speed index in seeds of the uvaieira (Eugenia pyriformis Cambess.) from different sources and at three levels of drying. $\mathrm{D}_{0}$ - non-dried seeds; $\mathrm{D}_{\mathrm{I}}-1$ st level of drying; $\mathrm{D}_{\mathrm{II}}$ - 2nd level of drying

\begin{tabular}{|c|c|c|c|}
\hline \multirow{2}{*}{$\begin{array}{l}\text { Source of material } \\
\text { (Water content for level of drying, } \mathrm{g} \mathrm{g}^{-1} \text { ) }\end{array}$} & \multicolumn{3}{|c|}{ Level of drying } \\
\hline & $\mathrm{D}_{0}$ & $\mathrm{D}_{\mathrm{I}}$ & $\mathrm{D}_{\mathrm{II}}$ \\
\hline $\operatorname{LAV} 1\left(\mathrm{D}_{0}=1.53 ; \mathrm{D}_{\mathrm{I}}=1.00 ; \mathrm{D}_{\mathrm{II}}=0.70\right)$ & $0.93 \mathrm{cdA}$ & $0.35 \mathrm{fgB}$ & 0.14 cdefC \\
\hline $\operatorname{LAV} 2\left(\mathrm{D}_{0}=1.12 ; \mathrm{D}_{\mathrm{I}}=1.00 ; \mathrm{D}_{\mathrm{II}}=0.67\right)$ & $1.09 \mathrm{bcA}$ & $1.21 \mathrm{bA}$ & $0.31 \mathrm{bcB}$ \\
\hline $\operatorname{SBS} 1\left(\mathrm{D}_{0}=1.87 ; \mathrm{D}_{\mathrm{I}}=1.17 ; \mathrm{D}_{\mathrm{II}}=0.67\right)$ & 0.70 efg $\mathrm{A}$ & $0.26 \mathrm{ghB}$ & 0.12 cdefC \\
\hline $\operatorname{SBS} 2\left(D_{0}=1.34 ; D_{I}=1.04 ; D_{I I}=0.64\right)$ & $0.86 \mathrm{de} A$ & $0.68 \mathrm{deB}$ & $0.30 \mathrm{bcdC}$ \\
\hline $\operatorname{SBS} 3\left(\mathrm{D}_{0}=1.12 ; \mathrm{D}_{\mathrm{I}}=0.96 ; \mathrm{D}_{\mathrm{II}}=0.64\right)$ & $0.82 \mathrm{de} A$ & $0.96 \mathrm{bcA}$ & $0.26 \mathrm{bcdeC}$ \\
\hline $\operatorname{CAM} 1\left(\mathrm{D}_{0}=1.58 ; \mathrm{D}_{\mathrm{I}}=0.96 ; \mathrm{D}_{\mathrm{II}}=0.79\right)$ & $0.81 \operatorname{def} \mathrm{A}$ & $0.38 \mathrm{fgB}$ & $0.06 \mathrm{fC}$ \\
\hline $\operatorname{CAM} 2\left(D_{0}=1.19 ; D_{I}=0.96 ; D_{I I}=0.64\right)$ & $1.22 \mathrm{bA}$ & $0.62 \mathrm{deB}$ & $0.21 \mathrm{bcdefC}$ \\
\hline $\operatorname{CAM} 3\left(\mathrm{D}_{0}=1.44 ; \mathrm{D}_{\mathrm{I}}=0.96 ; \mathrm{D}_{\mathrm{II}}=0.70\right)$ & $0.72 \operatorname{efg} \mathrm{A}$ & $0.25 \mathrm{ghB}$ & 0.31 cdefB \\
\hline $\operatorname{SPA} 1\left(\mathrm{D}_{0}=2.16 ; \mathrm{D}_{\mathrm{I}}=1.00 ; \mathrm{D}_{\mathrm{II}}=0.75\right)$ & 0.59 ghiA & $0.23 \mathrm{ghB}$ & 0.20 bcdefB \\
\hline $\operatorname{SPA} 2\left(\mathrm{D}_{0}=1.82 ; \mathrm{D}_{\mathrm{I}}=1.17 ; \mathrm{D}_{\mathrm{II}}=0.67\right)$ & $0.63 \mathrm{fghA}$ & $0.65 \mathrm{de} A$ & $0.37 \mathrm{abB}$ \\
\hline $\operatorname{SPA} 3\left(\mathrm{D}_{0}=1.90 ; \mathrm{D}_{\mathrm{I}}=1.08 ; \mathrm{D}_{\mathrm{II}}=0.70\right)$ & 0.70 efg $\mathrm{A}$ & $0.31 \mathrm{fgB}$ & 0.17 cdefB \\
\hline
\end{tabular}


Table 5 Continued

\begin{tabular}{|c|c|c|c|}
\hline IBI1 $\left(D_{0}=1.53 ; D_{I}=0.96 ; D_{I I}=0.64\right)$ & $0.89 \mathrm{de} A$ & $0.33 \mathrm{fgB}$ & 0.14 cdefC \\
\hline $\operatorname{IBI} 2\left(\mathrm{D}_{0}=1.71 ; \mathrm{D}_{\mathrm{I}}=1.22 ; \mathrm{D}_{\mathrm{II}}=0.85\right)$ & 0.73 efg A & $0.51 \mathrm{efB}$ & $0.40 \mathrm{abC}$ \\
\hline $\operatorname{SBC} 1\left(\mathrm{D}_{0}=1.27 ; \mathrm{D}_{\mathrm{I}}=1.04 ; \mathrm{D}_{\mathrm{II}}=0.75\right)$ & $0.87 \mathrm{de} A$ & $0.97 \mathrm{bcA}$ & $0.51 \mathrm{aB}$ \\
\hline $\operatorname{SBC} 2\left(D_{0}=1.38 ; D_{I}=1.08 ; D_{I I}=0.75\right)$ & $0.88 \mathrm{de} \mathrm{A}$ & $0.77 \mathrm{cdA}$ & $0.31 \mathrm{bcB}$ \\
\hline $\operatorname{SAA}\left(D_{0}=1.56 ; D_{I}=0.96 ; D_{I I}=0.67\right)$ & $0.42 \mathrm{iA}$ & $0.09 \mathrm{hB}$ & $0.08 \mathrm{efB}$ \\
\hline $\operatorname{JUM}\left(\mathrm{D}_{0}=1.47 ; \mathrm{D}_{\mathrm{I}}=1.00 ; \mathrm{D}_{\mathrm{II}}=0.69\right)$ & $1.42 \mathrm{aA}$ & $0.69 \mathrm{deB}$ & 0.10 defC \\
\hline $\operatorname{RIB}\left(\mathrm{D}_{0}=1.70 ; \mathrm{D}_{\mathrm{I}}=0.96 ; \mathrm{D}_{\mathrm{II}}=0.72\right)$ & $1.20 \mathrm{bA}$ & $1.40 \mathrm{aB}$ & $0.28 \mathrm{bcdC}$ \\
\hline $\operatorname{ITA}\left(\mathrm{D}_{0}=1.93 ; \mathrm{D}_{\mathrm{I}}=1.13 ; \mathrm{D}_{\mathrm{II}}=0.70\right)$ & $1.43 \mathrm{aA}$ & $1.02 \mathrm{bB}$ & 0.18 bcdefC \\
\hline $\operatorname{PAR}\left(\mathrm{D}_{0}=1.49 ; \mathrm{D}_{\mathrm{I}}=1.00 ; \mathrm{D}_{\mathrm{II}}=0.85\right)$ & 0.44 hiA & $0.23 \mathrm{ghB}$ & 0.11 cdefB \\
\hline $\mathrm{CV}(\%)$ & & 12.06 & \\
\hline
\end{tabular}

*Mean values followed by the same letter (lowercase compares source of material and uppercase compares levels of drying) do not differ by Tukey's test at $5 \%$

Table 6 - Simple correlation coefficients (r) between meteorological data and physical and physiological data in seeds of the uvaieira (Eugenia pyriformis Cambess.) from different sources

\begin{tabular}{|c|c|c|c|c|c|c|c|c|c|c|c|}
\hline & $\mathrm{DD}$ & Rain & $\mathrm{DM}$ & $\mathrm{GSD}_{\mathrm{I}}$ & $\mathrm{GSD}_{\mathrm{II}}$ & $\mathrm{GD}_{0}$ & $\mathrm{GD}_{\mathrm{L}}$ & $\mathrm{GD}_{\mathrm{II}}$ & $\mathrm{GSID}_{0}$ & GSID $_{\text {I }}$ & GSID $_{\mathrm{II}}$ \\
\hline Cycle & $0.60^{*}$ & $0.61^{*}$ & $0.53^{*}$ & -0.03 & -0.02 & -0.13 & -0.09 & 0.07 & -0.39 & 0.21 & -0.03 \\
\hline Min & $0.54 *$ & -0.07 & -0.26 & -0.43 & $-0.49 *$ & 0.13 & -0.26 & -0.33 & -0.26 & -0.38 & -0.39 \\
\hline Max & -0.02 & -0.40 & 0.03 & $0.46^{*}$ & 0.06 & 0.28 & 0.30 & -0.11 & $0.56^{*}$ & 0.15 & 0.03 \\
\hline $\mathrm{DD}$ & & 0.23 & 0.37 & 0.02 & -0.34 & 0.12 & 0.09 & -0.29 & -0.16 & 0.03 & -0.32 \\
\hline Rain & & & 0.16 & -0.05 & -0.03 & -0.30 & -0.13 & -0.11 & -0.13 & 0.03 & 0.06 \\
\hline WC & & & $-0.71 *$ & $-0.51 *$ & -0.37 & -0.27 & $-0.59 *$ & 0.43 & -0.01 & $-0.66^{*}$ & 0.29 \\
\hline $\mathrm{DM}$ & & & & 0.36 & 0.34 & 0.28 & $0.46^{*}$ & 0.36 & 0.01 & $0.51^{*}$ & 0.17 \\
\hline $\mathrm{GSD}_{0}$ & & & & 0.10 & -0.24 & $0.65^{*}$ & 0.07 & -0.32 & $0.46^{*}$ & -0.14 & $-0.46^{*}$ \\
\hline $\mathrm{GSD}_{\mathrm{I}}$ & & & & & $0.53^{*}$ & $0.80^{*}$ & $0.56^{*}$ & $0.65^{*}$ & $0.88^{*}$ & 0.05 & $0.65^{*}$ \\
\hline $\mathrm{GSD}_{\text {II }}$ & & & & & & -0.06 & $0.51^{*}$ & $0.87 *$ & 0.05 & $0.65^{*}$ & $0.88^{*}$ \\
\hline $\mathrm{GD}_{0}$ & & & & & & & 0.35 & 0.09 & $0.45^{*}$ & 0.09 & -0.29 \\
\hline $\mathrm{GD}_{\mathrm{I}}$ & & & & & & & & $0.61^{*}$ & $0.47^{*}$ & $0.88^{*}$ & $0.52 *$ \\
\hline $\mathrm{GD}_{\text {II }}$ & & & & & & & & & -0.02 & $0.71^{*}$ & $0.82 *$ \\
\hline $\mathrm{GSID}_{0}$ & & & & & & & & & & 0.33 & -0.01 \\
\hline GSID $_{\mathrm{I}}$ & & & & & & & & & & & $0.71 *$ \\
\hline
\end{tabular}

Cycle = maturation cycle; Min = mimimum temperature; Max = maximum temperature; $\mathrm{DD}=$ degree-days; Rain = accumulated rainfall; WC = water content; $\mathrm{DM}=$ dry matter; $\mathrm{GSD}_{0}=$ non-dried germinated seeds; $\mathrm{GSD}_{\mathrm{I}}=$ germinated seeds at the 1 st level of drying; $\mathrm{GSD}_{\mathrm{II}}=$ germinated seeds at the 2nd level of drying; $\mathrm{GD}_{0}=$ non-dried germination; $\mathrm{GD}_{\mathrm{I}}=$ germination at the 1 st level of drying; $\mathrm{GD}_{\mathrm{II}}=$ germination at the 2 nd level of drying; $\mathrm{GSID}_{0}=$ non-dried germination speed index; GSID $=$ germination speed index at the 1st level of drying; GSID $=$ germination speed index at the 2 st $_{\mathrm{II}}$ level of drying. $(*)=r$ significant at $5 \%$ probability. Data not shown, or with no asterisk, correspond to non-significant correlations

also influence the cycle of seed formation. This result is similar to those obtained by Lamarca et al. (2013a) for the same species. Considering that accumulated degree-days showed a wide variation, and therefore in this case did not define the time of fruit and seed dispersal, it can be assumed that the amount of rainfall has a significant influence on the maturation cycle of the seeds in the uvaieira. Having a positive correlation, the greater the rainfall, the longer the development cycle of the uvaieira seeds. However, this influence does not translate into extending the maturation process, since there was no correlation between accumulated rainfall 
and water content or dry matter content of the dispersed seeds (Table 6). On the other hand, the maximum and minimum temperatures alone were not correlated with the cycle, and therefore have little influence on it (Table 6). Although the maturation cycles of seeds are programmed genetically, comparing collections made in any one region (from the same parent plants in CAM, SPA, LAV, IBI and SBC), but at different periods, it is found that in years of high rainfall, the cycles were longer and, consequently, there was a greater accumulation of degree-days.

A general analysis of the results showed that the water and thermal conditions of the environment indeed have a great influence on the maturation of uvaieira seeds, both for the cycle and the final quality of the produced seeds, especially the level of desiccation tolerance. For Daws et al. (2004, 2006), Dussert et al. (2000) and Joët, Ourcival and Dussert (2013), variations in desiccation tolerance within populations of the same species are a result of environmental conditions, which may lead to early seed dispersal, that is before they complete maturation. This probably happened with the uvaieira seeds from the different sources, creating differences in the progress of the development and maturation process. Thus, desiccation tolerance in this case could progress further, for more favourable water and thermal conditions of the environment, resulting in a longer period of maturation. It is interesting to note that all the seeds that showed less than $50 \%$ germination (except for CAM3) or a GSI less than or equal to 0.20 after the second level of drying, were dispersed with a water content greater or equal to $1.50 \mathrm{~g} \mathrm{~g}^{-1}$ (60\% wet basis).

It is a fact that variations in the level of desiccation tolerance among the sources were enough to identify the influence of the environment on desiccation tolerance. Analysing the seeds from different altitudes at SBS for example, it can be seen that at higher altitudes, with lower air temperatures (maximum and minimum), the period of maturation was longer, increasing the total of degree-days. On average, for each 100-meter increase in altitude, the air temperature decrease by $0.6^{\circ} \mathrm{C}$ (PEREIRA;ANGELOCCI; SENTELHAS, 2002). This increase apparently affects the maturation cycle and the accumulation of degree-days. Seeds formed at these higher altitudes (SBS2 and SBS3) for example, were dispersed with a lower water content and greater quantity of dry matter, resulting in less sensitivity to desiccation. This suggests that the seeds with a higher water content at the time of dispersal were more sensitive to desiccation due to their lesser maturity, as suggested by Barbedo, Centeno and Figueiredo-Ribeiro (2013), and also verified during the maturation of desiccation-tolerant seeds (LEDUC et al., 2012; SCHWALLIER; BHOOPALAN; BLACKMAN, 2011.). When analysing the results for seeds from the same parent plants but in different years, this effect from maturity becomes clearer. The seeds from LAV, CAM and SPA for example, became more sensitive to desiccation the higher their water content at the time of dispersal, as seen from the values after the first and/or second level of drying.

The air temperature, represented by the concept of degree-days, shows a strong relationship to seed development and maturation, as well as the acquisition of desiccation tolerance. Daws et al. (2004, 2006) for example, found that regions that favoured the greatest accumulation of degree-days during formation saw the dispersal of seeds that were more vigorous and more tolerant to desiccation; such was not observed in this study. In this work, the lower the minimum temperature, or higher the maximum temperature, the more tolerant to desiccation the seeds became. In other words, the sum of degree-days seems to influence desiccation tolerance, but must be associated with a minimum in temperature range.

Finally, variations in the degree of desiccation tolerance between the regions and collection times of the uvaieira seeds are dependent on the source of the material. It should also be noted that the water and thermal conditions of the environment might influence the maturation cycle, as well as the physiological quality and acquisition of desiccation tolerance of the seeds. Desiccation tolerance in seeds of the uvaieira depends therefore on their degree of maturation at the time of dispersal, confirming the assertion of Barbedo, Centeno and Figueiredo-Ribeiro (2013) that the level of recalcitrance in the seeds depends on the point of physiological maturity at the time they detach from the mother plant.

\section{CONCLUSION}

Desiccation tolerance in seeds of the uvaieira depends on the stage of physiological maturity at the time of dispersal, which in turn depends on the water and thermal conditions of the environment during formation.

\section{ACKNOWLEDGEMENTS}

The authors wish to thank the Vale do Ribeira Regional Hub - Pariquera-açú (SP), the CATI at Itaberá (SP), the CATI at São Bento do Sapucaí (SP), the Casa da Agricultura in Jumirim (SP), USP at Ribeirão Preto (SP), the São Paulo Botanical Institute (SP), the Campinas Institute of Agronomy (SP) and the Federal University of Lavras (MG). Thanks also go to the Institute of Astronomy and Geophysics of the University of São Paulo for their permission in the collection and supply of meteorological data, to Dr Lucia Rossi (IBt) for the species identification, to 
CNPq and CAPES for the grants awarded to the lead author, to CNPq for the Research Productivity grant awarded to the last author, and for their financial assistance of the project.

\section{REFERENCES}

AMADOR, T. S.; BARBEDO, C. J. Potencial de inibição da regeneração de raízes e plântulas em sementes germinantes de Eugenia pyriformis. Pesquisa Agropecuária Brasileira, v. 46, n. 8, p. 814-821, 2011.

ANGELOVICI, R. et al. Seed desiccation: a bridge between maturation and germination. Trends in Plant Science, v. 15, n. 4, p. 211-218, 2010 .

ARAÚJO, E. C. de. et al. Efeito da dessecação e armazenamento sobre a qualidade fisiológica de sementes de Syzygium jambolanum Lam. Revista Ciência Agronômica, v. 39, n. 3, p. $455-462,2008$

BARBEDO, C. J.; CENTENO, D. C.; FIGUEIREDO-RIBEIRO, R. C. L. Do recalcitrant seeds really exist? Hoehnea, v. 40, n. 4, p. 583-593, 2013.

BRASIL. Ministério de Agricultura, Pecuária e Abastecimento. Regras para análises de sementes. Brasília, DF: MAPA: ACS, 2009. 399 p.

DAWS, M. I. et al. Developmental heat sum influences recalcitrant seed traits in Aesculus hippocastanum across Europe. New Phytologist, v. 162, n. 1, p.157-166, 2004.

DAWS, M. I. et al. Variable desiccation tolerance in Acer pseudoplatanus seeds in relation to developmental conditions: a case of phenotypic recalcitrance? Functional Plant Biology, v. 33, n. 1, p. 59-66, 2006.

DELGADO, L. F.; BARBEDO, C. J. Tolerância à dessecação de sementes de espécies de Eugenia. Pesquisa Agropecuária Brasileira, v. 42, n. 2, p. 265-272, 2007.

DELGADO, L. F.; BARBEDO, C. J. Water potential and viability of seeds of Eugenia (Myrtaceae), a tropical tree species, based upon different levels of drying. Brazilian Archives of Biology and Technology, v. 55, n. 4, p. 583-590, 2012.

DUSSERT, S. et al. Relationship between seed desiccation sensitivity, seed water content at maturity and climatic characteristics of native environments of nine Coffea L. species. Seed Science Research, v. 10, n. 3, p. 293-300, 2000.

HAY, F. R.; PROBERT, R. J. Advances in seed conservation of wild plant species: a review of recent research. Conservation Physiology, v. 1, n. 1, p. 1-11, 2013.

JOËT, T.; OURCIVAL, J. M.; DUSSERT, S. Ecological significance of seed desiccation sensitivity in Quercus ilex. Annals of Botany, v. 111, n. 4, p. 693-701, 2013.

LAMARCA, E. V. et al. Contribuições do conhecimento local sobre o uso de Eugenia spp. em sistemas de policultivos e agroflorestas. Revista Brasileira de Agroecologia, v. 8, n. 3, p. 119-130, 2013b.
LAMARCA, E. V.; SILVA, C. V.; BARBEDO, C. J. Limites térmicos para a germinação em função da origem de sementes de espécies de Eugenia (Myrtaceae) nativas do Brasil. Acta Botanica Brasilica, v. 25, n. 2, p. 293-300, 2011.

LAMARCA, E.V. et al. Maturation of Eugenia pyriformis seeds under different hydric and thermal conditions. Anais da Academia Brasileira de Ciências, v. 85, n. 1, p. 223-233, 2013a.

LEDUC, S. N. M. et al. Non-structural carbohydrates of immature seeds of Caesalpinia echinata (Leguminosae) are involved in the induction of desiccation tolerance. Australian Journal of Botany, v. 60, n. 1, p. 42-48, 2012.

MAGUIRE, J. D. Speed of germination-aid in selection and evaluation for seedling emergence and vigor. Crop Science, v. 2, n. 2, p. 176-177, 1962.

MARTINS, C. C. et al. Secagem e armazenamento de sementes de juçara. Revista Árvore, v. 33, n. 4, p. 635-642, 2009.

PEDRO JUNIOR, M. J. et al. Estimativa de graus-dia em função de altitude e latitude para o estado de São Paulo. Bragantia, v. 36, n. 5, p. 89-92, 1977.

PEREIRA, A. R.; ANGELOCCI, L. R.; SENTELHAS, P. C. Agrometeorologia: fundamentos e aplicações práticas. Guaíba: Livraria e Editora Agropecuária, 2002. 478 p.

PEREIRA, W. V. S. et al. Desiccation tolerance of Tapirira obtusa seeds collected from different environments. Revista Brasileira de Sementes, v. 34, n. 3, p. 388-396, 2012.

PÉREZ, H. E.; HILL, L. M.; WALTERS, C. An analysis of embryo development in palm: interactions between dry matter accumulation and water relations in Pritchardia remota (Arecaceae). Seed Science Research, v. 22, n. 2, p. 97-111, 2012

SANTANA, D. G.; RANAL, M. A. Análise da germinação: um enfoque estatístico. Brasília: Universidade de Brasília, 2004. 248 p.

SCALON, S. P. Q. et al. Sensibilidade à dessecação e ao armazenamento em sementes de Eugenia pyriformis Cambess. (uvaia). Revista Brasileira de Fruticultura, v. 34, n. 1, p. 269276, 2012.

SCHWALLIER, R.; BHOOPALAN, V.; BLACKMAN, S. The influence of seed maturation on desiccation tolerance in Phalaenopsis amabilis hybrids. Scientia Horticulturae, v. 128, n. 2, p. 136-140, 2011.

TEIXEIRA, C. C.; BARBEDO, C. J. The development of seedlings from fragments of monoembryonic seeds as an important survival strategy for Eugenia (Myrtaceae) tree species. Trees, Structure and Function, v. 26, n. 3 p. 1069-1077, 2012.

VILLA NOVA, N. A. et al. Estimativa de graus-dia acumulados acima de qualquer temperatura base em função das temperaturas máxima e mínima. Caderno Ciência da Terra, v. 30, n. 2, p. 1-8, 1972.

WALTERS, C. Levels of recalcitrance in seeds. Revista Brasileira de Fisiologia Vegetal, v. 12, p. 7-21, 2000. Edição especial. 